\title{
Electrostatic Surface Modes in Magnetized Quantum Electron-Hole Plasma
}

\author{
Bahaa F. Mohamed1, Abrar A. Salah ${ }^{2 *}$ \\ ${ }^{1}$ Plasma Physics Department, N.R.C., Egyptian Atomic Energy Authority, Cairo, Egypt \\ ${ }^{2}$ Physics Department, Faculty of Science, Ain-Shams University, Cairo, Egypt \\ Email: mohamedbahf@yahoo.co.uk, ^abrar.physics1989@gmail.com
}

How to cite this paper: Mohamed, B.F. and Salah, A.A. (2021) Electrostatic Surface Modes in Magnetized Quantum ElectronHole Plasma. Journal of Modern Physics, 12, 1518-1526.

https://doi.org/10.4236/jmp.2021.1211091

Received: February 19, 2020

Accepted: September 6, 2021

Published: September 9, 2021

Copyright (c) 2021 by author(s) and Scientific Research Publishing Inc. This work is licensed under the Creative Commons Attribution International License (CC BY 4.0).

http://creativecommons.org/licenses/by/4.0/

\begin{abstract}
The excitation of electrostatic surface waves on a semibounded quantum plasma-vacuum interface parallel to an applied magnetic field with electron-hole degeneracy is investigated. The wave equations of the electrostatic potential and both of the perturbed electron and hole plasma densities have been solved analytically. By using quantum hydrodynamic (QHD) model and the Poisson's equation with appropriate boundary conditions, the general dispersion relation of these surface modes has been obtained. It is also solved and studied numerically for different cases of plasmas (magnetized or unmagnetized, classical or quantum). We have found that the density ratio of hole-electron plasma plays essential role on the dispersion of the modes along the wavelength beside the quantum and magnetic field.
\end{abstract}

\section{Keywords}

Surface Plasma Waves, Beam-Plasma Interaction, Quantum Plasma

\section{Introduction}

In recent years, the plasmas physics has been widely studied and different research has been presented [1]-[6]. Recently, several studies of quantum plasmas have appeared in the literature [7]-[13]. Plasma can be regarded as quantum when the quantum nature of its particles significantly affects its macroscopic properties [14]. There has been a great interest in investigating physical properties of quantum plasmas since the quantum plasmas can be found in various nano-scale objects such as nano-wires, quantum dot, and semiconductor devices as well as in dense laser produced plasmas [15]. There are various models to study the quantum effects in plasma, for example, the Wigner-Poisson system which involves an integro-differential system and the popular QHD model. The 
QHD model can be considered as an extension of the usual fluid model of plasma [16]. The QHD includes the quantum forces involving Fermi electron temperature and quantum Bohm potential [17]. Several authors incorporated the effect of the dispersion caused by the quantum Bohm potential in the study of electrostatic plasma waves [18] [19]. Moradi A. [19] investigated the propagation of surface electrostatic oscillations on an electron quantum plasma half space, taking into account the quantum effects. Misra A. [20] studied the propagation of surface electromagnetic waves along a uniform magnetic field in a quantum electron-hole semiconductor plasma. He has shown that the surface modes to be significantly modified in the case of high-conductivity semiconductor plasmas where electrons and holes may be degenerate. Besides, a hydrodynamic model describing steady-state and dynamic electron and hole transport properties of graphene structures has been developed by Svintsov, D., et al. [21] which accounts for the features of the electron and hole spectra. They demonstrated its workability in some applications, in different structures and devices based on the standard semiconductors.

In this paper, we have investigated the propagation of electrostatic surface waves at the electron-hole plasma-vacuum interface parallel to an applied magnetic field. It has been considered the degeneracy of the electron-hole due to the quantum tunneling effect associated with the Bohm potential.

\section{Model of Equations}

Let us consider a Cartesian geometry where the plane $x=0$ separates the half-space $x>0$ filled by a homogeneous magnetized quantum plasma consisting of electrons and holes to be denoted respectively by $\alpha \quad(=e$ and $h)$ and vacuum $x<0$. Electrostatic surface waves are considered to propagate in completely degenerate dense plasma. So, the rate of particle collisions is limited due to the Pauli blocking mechanism which allows only degenerate particles with energies limited to a narrow range around the Fermi energy to interact, hence the plasma may be considered to be almost collisionless. We also assume that the electron and hole densities are, in general, not equal. In a uniform external magnetic field $\vec{B}=B_{0} \hat{z}$, The dynamics of such a plasma is governed by following quantum hydrodynamic equations:

$$
\begin{gathered}
\frac{\partial n_{\alpha}}{\partial t}+\operatorname{div}\left(n_{\alpha} \vec{V}_{\alpha}\right)=0 \\
m_{e} \frac{\mathrm{d} \vec{v}_{e}}{\mathrm{~d} t}=e \vec{\nabla} \varphi-\frac{e}{c}\left[\vec{v}_{e} \times\left(\vec{B}+\vec{B}_{0}\right)\right]-\frac{\vec{\nabla} P_{e}}{n_{e}}+\frac{\hbar^{2}}{2 m_{e}} \vec{\nabla}\left(\frac{\nabla^{2} \sqrt{n_{e}}}{\sqrt{n_{e}}}\right) \\
m_{h} \frac{\mathrm{d} \vec{v}_{h}}{\mathrm{~d} t}=-e \vec{\nabla} \varphi+\frac{e}{c}\left[\vec{v}_{h} \times\left(\vec{B}+\vec{B}_{0}\right)\right]-\frac{\vec{\nabla} P_{h}}{n_{h}}+\frac{\hbar^{2}}{2 m_{h}} \vec{\nabla}\left(\frac{\nabla^{2} \sqrt{n_{h}}}{\sqrt{n_{h}}}\right) \\
\nabla^{2} \varphi=4 \pi e\left(n_{e}-n_{h}\right)
\end{gathered}
$$

The symbols here have their conventional meaning. Note that the degenerate 
electron and hole pressure depend only on the electron and hole number density but not on their temperature. We now use the following normalization of the number density $n_{\alpha}$ and the velocity $v_{\alpha}$ for $\alpha$-species are normalized by equilibrium value $n_{\alpha o}$ and $c_{s}, n_{\alpha} \rightarrow n_{\alpha} / n_{\alpha 0}, \vec{v}_{\alpha} \rightarrow \vec{v}_{\alpha} / c_{s}$, (as in ref. [19]). Here, $c_{s}\left(=\sqrt{2 k_{B} T_{F e} / m_{h}}\right)$ is the Fermi velocity for relatively dense (electrons and holes) plasma. Moreover, the space and the time variables are normalized by $\omega_{p h} / c_{s}$ and $\omega_{p h}$ respectively. Also, the space derivative of the potential is normalized by $-m_{h} c_{s} \omega_{p h} / e$, with $\omega_{p \alpha}=\sqrt{n_{\alpha o} e^{2} / \varepsilon_{o} m_{\alpha}}$ is the plasma frequency and $\omega_{c \alpha}=e B_{0} / m_{\alpha} \omega_{p h}$ is the normalized cyclotron frequency. $m=m_{e} / m_{h}$ is the electron to hole mass ratio and $\delta=n_{h o} / n_{e o}$.

By considering that, the basic Equations (1)-(4) can be rewritten in the following normalized form:

$$
\begin{gathered}
\frac{\partial n_{\alpha}}{\partial t}+\operatorname{div} \vec{v}_{\alpha}=0 \\
m \frac{\partial \vec{v}_{e}}{\partial t}=\vec{\nabla} \varphi-\omega_{c h}\left(\vec{v}_{e} \times \vec{e}_{z}\right)-\gamma \vec{\nabla} n_{e}+\frac{H^{2}}{4} \vec{\nabla} \nabla^{2} n_{e} \\
\frac{\partial \vec{v}_{h}}{\partial t}=-\vec{\nabla} \varphi+\omega_{c h}\left(\vec{v}_{h} \times \vec{e}_{z}\right)-\sigma \gamma \vec{\nabla} n_{e}+\frac{m H^{2}}{4} \vec{\nabla} \nabla^{2} n_{h} \\
\nabla^{2} \varphi=n_{e} / \delta-n_{h}
\end{gathered}
$$

where, $H=\sqrt{\delta} \hbar \omega_{p e} / 2 k_{B} T_{F e}$ is quantum coupling parameter and $\sigma=T_{F h} / T_{F e}\left(=\delta^{2 / 3} \mathrm{~m}\right)$ is the hole-electron temperature ratio related to the density ratio in which electrons and holes are degenerate with $\gamma=1 / 3$ (for the Fermi pressure).

By assuming that the amplitude of oscillation are small and any perturbed variable depends as $\psi=\psi(x) \exp \left(k_{y} \vec{e}_{y}-i \omega t\right)$, we can linearize and solve the system of Equations (5)-(8). Thus, we obtain the following wave equations for the perturbed electron density, hole density and electrostatic potential:

$$
\begin{gathered}
\frac{\partial^{2} n_{e}}{\partial x^{2}}-\frac{k_{y}^{2}}{Q}\left[Q-\frac{m^{2} \omega^{2}-\omega_{c h}^{2}}{m \gamma k_{y}^{2}}\right] n_{e}=\frac{1}{\gamma Q}\left[\frac{\partial^{2} \phi}{\partial x^{2}}-k_{y}^{2} \phi\right] \\
\frac{\partial^{2} n_{h}}{\partial x^{2}}-\frac{k_{y}^{2}}{Q^{\prime}}\left[Q^{\prime}-\frac{\omega^{2}-\omega_{c h}^{2}}{\sigma \gamma k_{y}^{2}}\right] n_{h}=\frac{1}{\sigma \gamma Q^{\prime}}\left[\frac{\partial^{2} \phi}{\partial x^{2}}-k_{y}^{2} \phi\right] \\
\frac{\partial^{2} \phi}{\partial x^{2}}-k_{y}^{2} \phi=\frac{n_{e}}{\delta}-n_{h}
\end{gathered}
$$

with $Q=1+\frac{H^{2} k_{y}^{2}}{4 \gamma}$ and $Q^{\prime}=1+\frac{m H^{2} k_{y}^{2}}{4 \gamma \sigma}$.

Thus, by taking into account the very slow nonlocal variation (i.e., $\left.k_{y}^{-2}\left(\partial^{2} / \partial x^{2}\right) \ll \partial^{2} / \partial x^{2} \ll k_{y}^{2}\right)$, the Equations (9)-(11) give the following wave equations of the perturbed densities of the beam and plasma:

$$
\frac{\partial^{2} n_{\alpha}}{\partial x^{2}}-\beta^{2} n_{\alpha}=0
$$


where,

$$
\begin{gathered}
\beta^{2}=\frac{\aleph^{2} \aleph^{\prime 2}-\left(1 / \sigma \delta \gamma^{2} Q Q^{\prime}\right)}{\aleph^{2}+\aleph^{\prime 2}} \\
\aleph^{2}=\frac{k_{y}^{2}}{Q}\left[Q-\frac{m^{2} \omega^{2}-\omega_{c h}^{2}}{m \gamma k_{y}^{2}}+\frac{1}{\delta \gamma k_{y}^{2}}\right], \aleph^{\prime 2}=\frac{k_{y}^{2}}{Q^{\prime}}\left[Q^{\prime}-\frac{\omega^{2}-\omega_{c h}^{2}}{\sigma \gamma k_{y}^{2}}+\frac{1}{\sigma \gamma k_{y}^{2}}\right]
\end{gathered}
$$

It is noted that the surface waves can only excited under the condition

$$
\left[1+\sigma \gamma Q^{\prime} k_{y}^{2}-\omega^{2}+\omega_{c h}^{2}\right]\left[1+\delta \gamma Q k_{y}^{2}-\frac{\delta}{m}\left(m^{2} \omega^{2}-\omega_{c e}^{2}\right)\right]>1
$$

\section{Dispersion Relations of Electrostatic Surface Modes}

In what follows, we will find solutions that represent the surface waves propagating along the interface $x=0$. To this end, Equation (12) has the following finite solution inside the quantum magnetized plasma:

$$
n_{\alpha}=A_{\alpha} \exp (-\beta x), \text { for } x \geq 0
$$

where, $A_{\alpha}$ is an arbitrary constant. Now, it is possible to obtain the electrostatic potential by solving the wave Equation (11):

$$
\begin{gathered}
\varphi=\varphi_{v} \exp \left(k_{y} x\right), \text { for } x \leq 0 \\
\varphi=\varphi_{\nu} \exp \left(-k_{y} x\right)+\frac{A_{e} / \delta-A_{h}}{\beta^{2}-k_{y}^{2}}\left[\exp (-\beta x)-\exp \left(-k_{y} x\right)\right], \text { for } x \geq 0
\end{gathered}
$$

$\varphi_{v}$ is the amplitude of the electrostatic potential in vacuum. The above Solutions (13)-(15) have been obtained by applying the boundary conditions at interface $(x=0)$ :

$$
\left.\varphi^{(\text {plasma })}(x)\right|_{x=0}=\left.\varphi^{(\text {vacuum })}(x)\right|_{x=0} \text { and }\left.\frac{\partial}{\partial x} \varphi^{(\text {plasma })}(x)\right|_{x=0}=\left.\frac{\partial}{\partial x} \varphi^{(\text {vacuum })}(x)\right|_{x=0}
$$

Beside that $v_{e x}=v_{h x}=0$ at $x=0$, it can be obtained the amplitudes of the perturbed electron and hole densities as follows:

$$
A_{e}=\frac{\alpha_{2}}{\alpha_{1}} \varphi_{v} \text { and } A_{h}=\frac{\alpha_{4}}{\alpha_{3}} \varphi_{v}
$$

where,

$$
\begin{aligned}
& \alpha_{1}=\gamma \omega_{c h} k_{y}\left[1-\frac{H^{2}}{4 \gamma}\left(\beta^{2}-k_{y}^{2}\right)\right]-m \gamma \omega \beta Q, \alpha_{2}=-k_{y}\left(m \omega+\omega_{c h}\right) \\
& \alpha_{3}=-\gamma \sigma \omega_{c h} k_{y}\left[1-\frac{m H^{2}}{4 \gamma \sigma}\left(\beta^{2}-k_{y}^{2}\right)\right]-\gamma \sigma \omega \beta Q^{\prime}, \quad \alpha_{4}=k_{y}\left(\omega-\omega_{c h}\right)
\end{aligned}
$$

It has also been obtained the following dispersion relations of the electrostatic surface modes on degenerate plasma:

$$
2 k_{y}\left(\beta+k_{y}\right) \alpha_{1} \alpha_{3}-\left(\alpha_{1} \alpha_{4}+\alpha_{2} \alpha_{3}\right)=0
$$




\section{Numerical Analysis and Discussions}

From the first sight, it is clear that the dispersion of the electrostatic modes (Equation (17)) depends on a lot of parameters (e.g., $\left.\omega_{c h}, H, \delta, \cdots\right)$. So, in this section, we are going to study the above dispersion relations (17) numerically for different cases, magnetized or unmagnetized, classical or quantum plasma (with the parameters $\gamma=1 / 3, m=0.25$ and $\sigma=1$ ).

First, the simplest form of the dispersion relation (17), in the case of the classical unmagnetized plasma, has two solutions (Figure 1). It is noticed that they have a starting common frequency $\left(\omega \approx 1.4 \omega_{p h}\right)$ of electrostatic oscillation at $k_{z}=0$.

In another case, unmagnetized quantum plasma, one of the solutions of the Equation (17) is studied in the Figure 2 with different electron-hole density ratios. It is found that as the hole density is increased with respect to the electron density, the frequency of the surface mode has been increased with respect to the hole plasma frequency along the wave number but the phase velocity of the modes still unchanged for different densities ratios ( $\delta=0.1,0.3,0.5,0.9$ ).

The other case concerning the classical magnetized plasma is also investigated through two solutions of Equation (17) in Figure 3 for different magnetic fields $\omega_{c h}=e B_{0} / m_{h} \omega_{p h}$. The first solution, Figure 3(a) investigates the dispersion of low frequency electrostatic surface modes $\left(\omega<\omega_{p h}\right)$. It is found that these modes have been excited in the beginning with $k_{y}=0$ and $\omega=0$ for different magnetic fields $\left(\omega_{c h}=0.1,0.3,0.5,0.9\right)$ and the phase velocity of the mode increased with increasing the external magnetic field.

On the other hand, the second solution, Figure 3(b), displays the dispersion of high frequency surface modes $\left(\omega>\omega_{p h}\right)$. It investigates that excitation of the

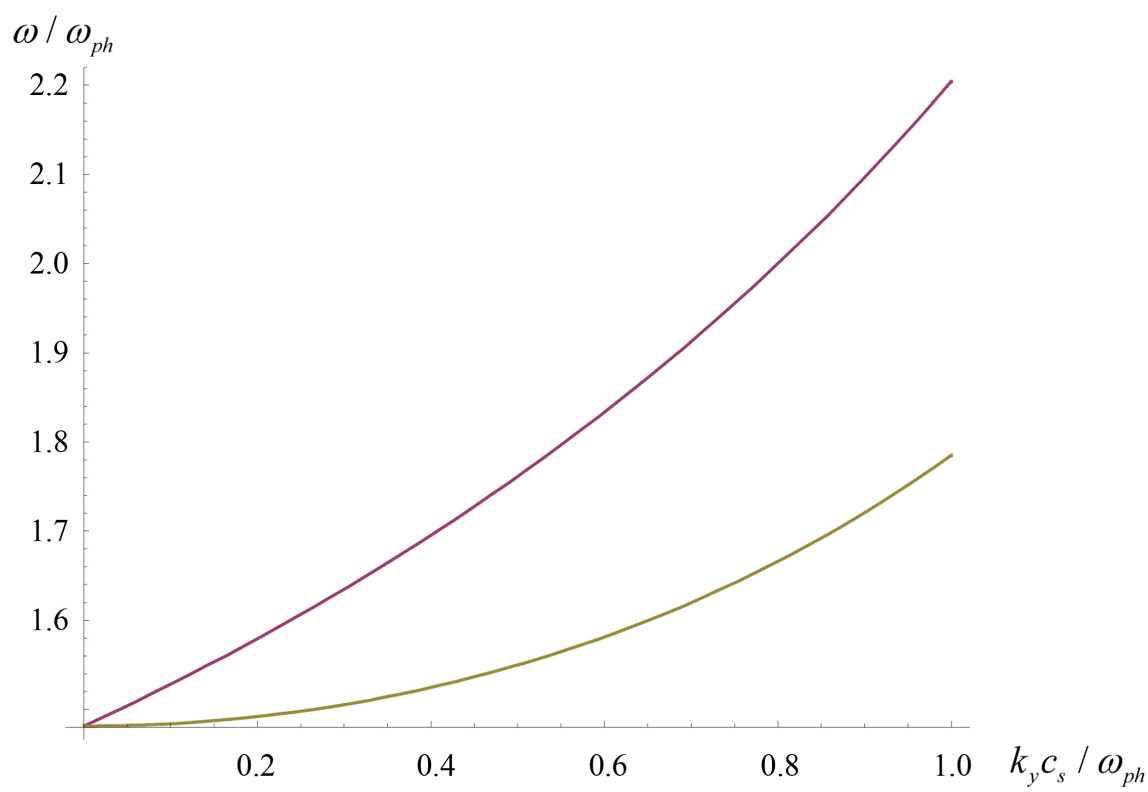

Figure 1. Dispersion relation of electrostatic surface modes for density ratios $(\delta=0.1)$ in unmagnetized electron-hole classical plasma $\left(\omega_{c h}=0, H=0\right)$. 


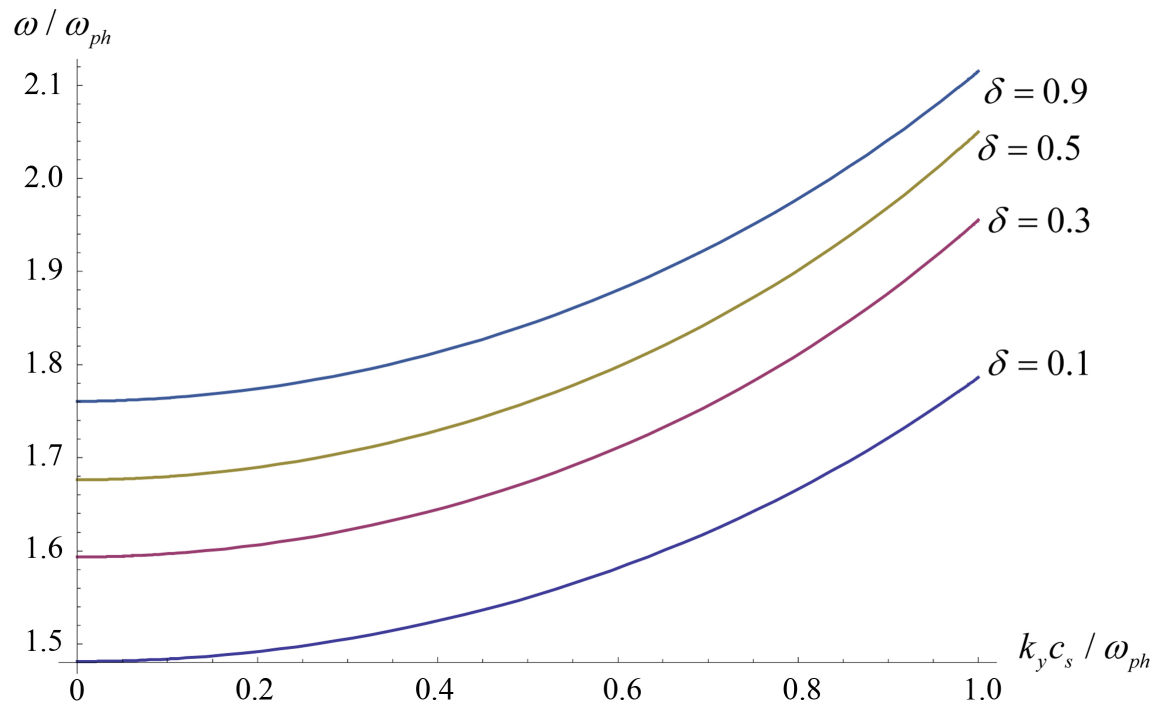

Figure 2. Dispersion relation of electrostatic surface modes for different density ratios ( $\delta=0.1,0.3,0.5,0.9)$ in unmagnetized quantum plasma ( $\omega_{c h}=0, H=0.1$ ).



(a)

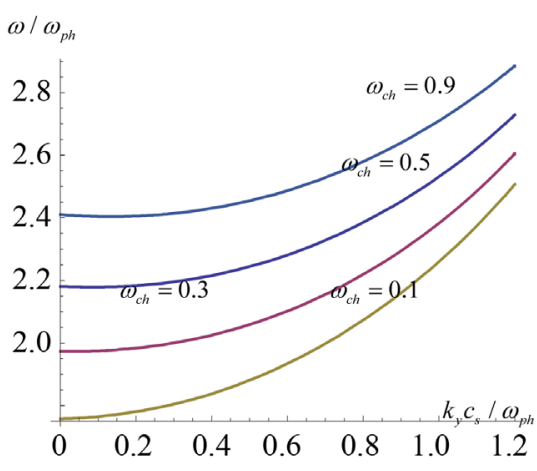

(b)

Figure 3. Two dispersion relations of the surface modes for different magnetic fields $\left(\omega_{c h}=0.1,0.3,0.5,0.9\right)$ in classical plasma $(H=0)$ with electron-hole density ratio $(\delta=0.1)$.

modes started at $k_{y}=0$ with different frequencies for different values of magnetic field and their phase velocity decreased with the increasing the intensity of magnetic field.

Figure 4 studied the case of unmagnetized quantum plasma with different quantum ratios $(H=0.1,0.3,0.5,0.7,0.9)$. It has been noticed that the excitation of the surface modes started with frequency emerge at $\omega \approx 1.5 \omega_{p h}$ for all quantum effect parameters. The dispersion of these modes indicates that phase velocity during the propagation increases faster to infinity with increasing the quantum effect.

Finally, the general case of the excitation of electrostatic surface modes in magnetized quantum plasma is investigated in Figure 5 with $\omega_{c h}=0.1$ and $\delta=0.1$. It is clear that as the quantum effect increased the phase velocity decreases for small quantum effect and its sign is changed with large quantum ratio $H$. 


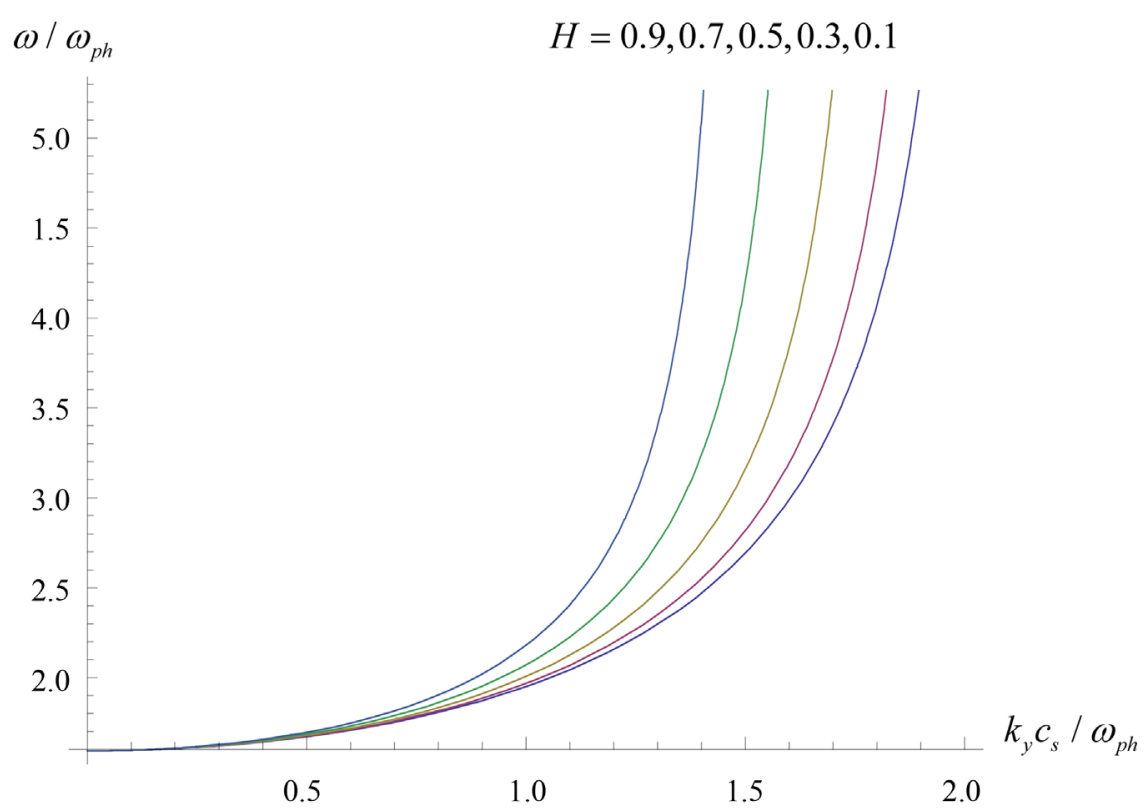

Figure 4. Dispersion relation of the electrostatic surface modes for different quantum ratios $(H=0.1,0.3,0.5,0.7,0.9)$ in unmagnetized quantum plasma with electron-hole density ratio $(\delta=0.3)$.

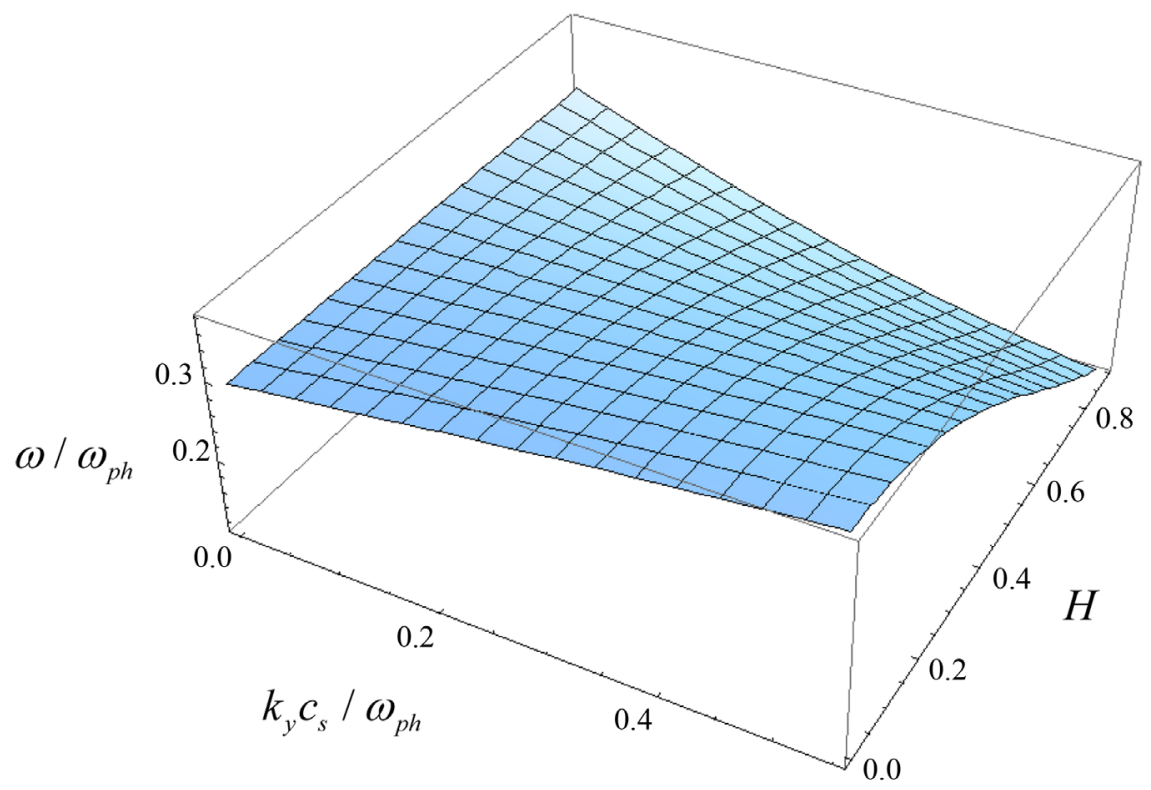

Figure 5. Dispersion relation of electrostatic surface modes with variation of quantum effect in magnetized electron-hole plasma with $\omega_{c h}=0.1$ and $\delta=0.1$.

\section{Conclusion}

In this work, the dispersion properties of the excitation of electrostatic surface waves with degenerate electron-hole plasma are studied by using the quantum hydrodynamic equations. The quantum effects due to Bohm potential are taken into account. We have obtained an analytical expression of the dispersion relation for the surface wave oscillations with normalized parameters. It has been 
solved numerically in different cases, magnetized or unmagnetized, classical or quantum plasmas. It is found that the quantum effects and the external magnetic field play a significant role on the dispersion of surface plasma modes where plasma energies of the system significantly modified. Besides, we have shown that the increase of electron-hole density make shift increase for the frequencies emerge.

\section{Conflicts of Interest}

The authors declare no conflicts of interest regarding the publication of this paper.

\section{References}

[1] Yang, X.-F., et al. (2011) Communications in Theoretical Physics, 56, 769. https://doi.org/10.1088/0253-6102/56/4/29

[2] Moradi, A. (2015) Physics of Plasmas, 22, Article ID: 014501. https://doi.org/10.1063/1.4906054

[3] Hussain, S. and Mahmood, S. (2011) Physics of Plasmas, 18, Article ID: 082109. https://doi.org/10.1063/1.3626556

[4] El-Labany, S., et al. (2010) Physics Letters A, 374, 960-964. https://doi.org/10.1016/j.physleta.2009.12.027

[5] Chatterjee, P., et al. (2009) Physics of Plasmas, 16, Article ID: 112106. https://doi.org/10.1063/1.3263695

[6] Khan, S. and Masood, W. (2008) Physics of Plasmas, 15, Article ID: 062301. https://doi.org/10.1063/1.2920273

[7] Brodin, G., Marklund, M. and Manfredi, G. (2008) Physical Review Letters, 100, Article ID: 175001. https://doi.org/10.1103/PhysRevLett.100.175001

[8] Haas, F. (2005) Physics of Plasmas, 12, Article ID: 062117. https://doi.org/10.1063/1.1939947

[9] Manfredi, G. (2005) Fields Institute Communications, 46, 263-287. https://doi.org/10.1090/fic/046/10

[10] Marklund, M., Eliasson, B. and Shukla, P.K. (2007) Physical Review E, 76, Article ID: 067401. https://doi.org/10.1103/PhysRevE.76.067401

[11] Shukla, P.K. and Eliasson, B. (2010) Physics-Uspekhi, 53, 51. https://doi.org/10.3367/UFNe.0180.201001b.0055

[12] Mahajan, S.M. and Asenjo, F.A. (2013) International Journal of Theoretical Physics, 54, 1435-1449.

[13] Zobaer, M., Roy, N. and Mamun, A. (2012) Journal of Modern Physics, 3, 604. https://doi.org/10.4236/jmp.2012.37082

[14] Vladimirov, S. and Tyshetskiy, Y.O. (2011) On Description of Quantum Plasma. https://doi.org/10.1063/1.3679592

[15] Chang, I.-S. and Jung, Y.-D. (2008) Physics Letters A, 372, 1498-1500. https://doi.org/10.1016/j.physleta.2007.10.004

[16] Sahu, B. (2011) Pramana, 76, 933-944. https://doi.org/10.1007/s12043-011-0072-9

[17] Mohamed, B.F. (2010) Physica Scripta, 82, Article ID: 065502. https://doi.org/10.1088/0031-8949/82/06/065502 
[18] Shukla, P. (2007) Physics Letters A, 369, 312-314.

https://doi.org/10.1016/j.physleta.2007.04.091

[19] Moradi, A. (2015) Physics of Plasmas, 22, Article ID: 014501. https://doi.org/10.1063/1.4906054

[20] Misra, A. (2011) Physical Review E, 83, Article ID: 057401. https://doi.org/10.1103/PhysRevE.83.057401

[21] Svintsov, D., et al. (2012) Journal of Applied Physics, 111, Article ID: 083715. https://doi.org/10.1063/1.4705382 